\title{
Asymptotic Solutions of Singular Perturbed Problems with an Instable Spectrum of the Limiting Operator
}

\author{
Burkhan T. Kalimbetov, Marat A. Temirbekov, \\ and Zhanibek O. Khabibullayev
}

Department of Mathematics, A. Yasawi International Kazakh-Turkish University, Turkestan 161200, Kazakhstan

Correspondence should be addressed to Marat A. Temirbekov, mtemirbekov@bk.ru

Received 2 March 2012; Accepted 3 April 2012

Academic Editor: Allaberen Ashyralyev

Copyright (C) 2012 Burkhan T. Kalimbetov et al. This is an open access article distributed under the Creative Commons Attribution License, which permits unrestricted use, distribution, and reproduction in any medium, provided the original work is properly cited.

\begin{abstract}
The regularization method is applied for the construction of algorithm for an asymptotical solution for linear singular perturbed systems with the irreversible limit operator. The main idea of this method is based on the analysis of dual singular points of investigated equations and passage in the space of the larger dimension, what reduces to study of systems of first-order partial differential equations with incomplete initial data.
\end{abstract}

\section{Introduction}

The investigation of singular perturbed systems for ordinary and partial differential equations occurring in systems with slow and fast variables, chemical kinetics, the mathematical theory of boundary layer, control with application of geoinformational technologies, quantum mechanics, and plasma physics (the Samarsky-Ionkin problem) has been studied by many researchers (see, e.g., [1-19]).

In this work, the algorithm for construction of an asymptotical solution for linear singular perturbed systems with the irreversible limit operator is given-the regularization method [1]. The main idea of this method is based on the analysis of dual singular points of investigated equations and passage in the space of the larger dimension, what reduces to the study of systems of first-order partial differential equations with incomplete (more exactly, point) initial data. 
In this paper, we consider linear singular perturbed systems in the form

$$
\varepsilon \dot{y}=A(t) y+h(t), \quad y(0, \varepsilon)=y^{0}, \quad t \in[0, T],
$$

where $y=\left\{y_{1}, \ldots, y_{n}\right\}, A(t)$ is a matrix of order $(n \times n), h(t)=\left\{h_{1}, \ldots, h_{n}\right\}$ is a known function, $y^{0} \in C^{n}$ is a constant vector, and $\varepsilon>0$ is a small parameter, in the case of violation of stability of a spectrum $\left\{\lambda_{j}(t)\right\}$ of the limiting operator $A(t)$.

Difference of such type problems from similar problems with a stable spectrum (i.e., in the case of $\lambda_{i}(t) \neq 0, \lambda_{i}(t) \neq \lambda_{j}(t), i \neq j, i, j=\overline{1, n}$ for all $\left.t \in[0, T]\right)$ is that the limiting system $0=A(t) \bar{y}+h(t)$ at violation of stability of the spectrum can have either no solutions or uncountable set of them. In the last case, presence of discontinuous on the segment $[0, T]$ solutions $\bar{y}(t)$ of the limiting system is not excluded. Under conditions, one can prove (see, e.g., $[1,6])$ that the exact solution $y(t, \varepsilon)$ of problem (1.1) tends (at $\varepsilon \rightarrow+0)$ to a smooth solution of the limiting system. However, there is a problematic problem about construction of an asymptotic solution of problem (1.1). When the spectrum is instable, essentially special singularities are arising in the solution of system (1.1). These singularities are not selected by the spectrum $\left\{\lambda_{j}(t)\right\}$ of the limiting operator $A(t)$. As it was shown in [3-7], they were induced by instability points $t_{j}$ of the spectrum.

In the present work, the algorithm of regularization method [1] is generalized on singular perturbed systems of the form (1.1), the limiting operator of which has some instable points of the spectrum. In order to construct the spectrum, we use the new algorithm requiring more constructive theory of solvability of iterative problems. These problems arose in application of the algorithm.

We will consider the problem (1.1) at the following conditions. Assume that

(i) $A(t) \in C^{\infty}\left([0, T], C^{n}\right), h(t) \in C^{\infty}[0, T]$; for any $t \in[0, T]$, the spectrum $\left\{\lambda_{j}(t)\right\}$ of the operator $A(t)$ satisfies the conditions:

(ii) $\lambda_{i}(t)=-\left(t-t_{i}\right)^{s_{i}} k_{i}(t), k_{i}(t) \neq 0, t_{i} \in[0, T], i=\overline{1, m}, m<n$ (here $s_{i}$ - are even natural numbers),

(iii) $\lambda_{i}(t) \neq 0, j=\overline{m+1, n}$,

(iv) $\lambda_{i}(t) \neq \lambda_{j}(t), i \neq j, i, j=\overline{1, n}$,

(v) $\operatorname{Re} \lambda_{j}(t) \leqslant 0, j=\overline{1, n}$.

\section{Regularization of the Problem}

We introduce basic regularized variables by the spectrum of the limiting operator

$$
\tau_{j}=\varepsilon^{-1} \int_{0}^{t} \lambda_{j}(s) d s \equiv \frac{\varphi_{j}(t)}{\varepsilon}, \quad j=\overline{1, n}
$$

Instable points $t_{i} \in[0, T]$ of the spectrum $\left\{\lambda_{j}(t)\right\}$ induce additional regularized variables described by the formulas

$$
\sigma_{i q_{i}}=e^{\varphi_{i}(t) / \varepsilon} \int_{0}^{t} e^{-\varphi_{i}(s) / \varepsilon} \frac{\left(s-t_{i}\right)^{q_{i}}}{q_{i}} d s \equiv \psi_{i q_{i}}(t, \varepsilon), \quad i=\overline{1, m}, q_{i}=\overline{0, s_{i}-1} .
$$


We consider a vector function $\tilde{y}(t, \tau, \sigma, \varepsilon)$ instead of the solution $y(t, \varepsilon)$ to be found for problem (1.1). This vector function is such that

$$
\left.\widetilde{y}(t, \tau, \sigma, \varepsilon)\right|_{\tau=\varphi, \sigma=\psi} \equiv y(t, \varepsilon)
$$

For $\tilde{y}(t, \tau, \sigma, \varepsilon)$, it is natural to set the following problem:

$$
\begin{aligned}
L_{\varepsilon} \tilde{y}(t, \tau, \sigma, \varepsilon) \equiv & \varepsilon \frac{\partial \tilde{y}}{\partial t}+\sum_{j=1}^{n} \lambda_{j}(t) \frac{\partial \tilde{y}}{\partial \tau_{j}}+\sum_{i=1}^{m} \sum_{q_{i}=0}^{s_{i}-1}\left[\lambda_{i}(t) \sigma_{i q_{i}}+\varepsilon \frac{\left(t-t_{i}\right)^{q_{i}}}{q_{i} !}\right] \frac{\partial \tilde{y}}{\partial \sigma_{i q_{i}}} \\
& -A(t) \tilde{y}=h(t), \quad \tilde{y}(0,0,0, \varepsilon)=y^{0} .
\end{aligned}
$$

We determine the solution of problem (2.4) in the form of a series

$$
\tilde{y}(t, \tau, \sigma, \varepsilon)=\sum_{k=-1}^{\infty} \varepsilon^{k} y_{k}(t, \tau, \sigma)
$$

with coefficients $y_{k}(t, \tau, \sigma) \in C^{\infty}[0, T]$.

If we substitute (2.5) in (2.4) and equate coefficients at identical degrees of $\varepsilon$, we obtain the systems for coefficients $y_{k}(t, \tau, \sigma)$ :

$$
\begin{gathered}
L y_{-1}(t, \tau, \sigma) \equiv \sum_{j=1}^{n} \lambda_{j}(t) \frac{\partial y_{-1}}{\partial \tau_{j}}+\sum_{i=1}^{m} \sum_{q_{i}=0}^{s_{i}-1} \lambda_{i}(t) \sigma_{i q_{i}} \frac{\partial y_{-1}}{\partial \sigma_{i q_{i}}}-A(t) y_{-1}=0, \quad y_{-1}(0,0,0)=0, \quad\left(\varepsilon^{-1}\right) \\
L y_{0}(t, \tau, \sigma)=-\frac{\partial y_{-1}}{\partial t}-\sum_{i=1}^{m} \sum_{q_{i}=0}^{s_{i}-1} \frac{\left(t-t_{i}\right)^{q_{i}}}{q_{i} !} \frac{\partial y_{-1}}{\partial \sigma_{i q_{i}}}+h(t), \quad y_{0}(0,0,0)=y^{0}, \\
\vdots \\
L y_{k+1}(t, \tau, \sigma)=-\frac{\partial y_{k}}{\partial t}-\sum_{i=1}^{m} \sum_{q_{i}=0}^{s_{i}-1} \frac{\left(t-t_{i}\right)^{q_{i}}}{q_{i} !} \frac{\partial y_{k}}{\partial \sigma_{i q_{i}}}, \quad k \geqslant 1, y_{k+1}(0,0,0)=0,
\end{gathered}
$$

\section{Resolvability of Iterative Problems}

We solve each of the iterative problems $\left(\varepsilon^{k}\right)$ in the following space of functions:

$$
\begin{aligned}
U=\{ & y(t, \tau, \sigma): y=\sum_{k=1}^{n} \sum_{j=1}^{n} y_{k j}(t) c_{k}(t) e^{\tau_{j}}+\sum_{k=1}^{n} \sum_{i=1}^{m} \sum_{q_{i}=0}^{s_{i}-1} y_{k i q_{i}}(t) c_{k}(t) \sigma_{i q_{i}} \\
& \left.+\sum_{k=1}^{n} y_{k}(t) c_{k}(t), \quad y_{k j}(t), y_{k i q_{i}}(t), y_{k}(t) \in C^{\infty}\left([0, T], C^{1}\right)\right\},
\end{aligned}
$$


where $c_{k}(t)$ are eigenvectors of the operator $A(t)$ corresponding eigenvalues $\lambda_{k}(t), k=\overline{1, n}$. We represent $U$ in the form of $U^{(1)} \oplus U^{(0)}$ where

$$
\begin{gathered}
U^{(0)}=\left\{y^{(0)}(t): y^{(0)}=\sum_{j=1}^{n} y_{j}^{(0)}(t) c_{j}(t), y_{j}^{(0)}(t) \in C^{\infty}\left([0, T], C^{1}\right)\right\}, \\
U^{(1)}=\frac{U}{U^{(0)}} .
\end{gathered}
$$

It is easy to note that each of the systems $\left(\varepsilon^{k+1}\right)$ can be written in the form

$$
\operatorname{Ly}(t, \tau, \sigma)=h(t, \tau, \sigma)
$$

where $h(t, \tau, \sigma)$ are the corresponding right hand side. Using representations of space $U$, we can write system (3.3) in the equivalent form

$$
\begin{aligned}
& \operatorname{Ly}^{(1)}(t, \tau, \sigma)=h^{(1)}(t, \tau, \sigma), \\
& -A(t) y^{(0)}(t)=h^{(0)}(t),
\end{aligned}
$$

where $y^{(1)}(t, \tau, \sigma), h^{(1)}(t, \tau, \sigma) \in U^{(1)}, y^{(0)}(t), h^{(0)}(t) \in U^{(0)}$.

We have the following result.

Theorem 3.1. Let $h^{(1)}(t, \tau, \sigma) \in U^{(1)}$ and satisfy conditions (i)-(iv). Then, system (3.4) is solvable in the $U^{(1)}$ if and only if

$$
\begin{gathered}
\left\langle h^{(1)}(t, \tau, \sigma), v_{j}(t, \tau, \sigma)\right\rangle \equiv 0 \quad \forall t \in[0, T], j=\overline{1, n} \\
\left\langle h^{(1)}(t, \tau, \sigma), v_{i q_{i}}(t, \tau, \sigma)\right\rangle \equiv 0, \quad i=\overline{1, m}, \quad q_{i}=\overline{0, s_{i}-1}
\end{gathered}
$$

where $v_{j}(t, \tau, \sigma), v_{i q_{i}}(t, \tau, \sigma)$ are basic elements of the kernel of the operator

$$
L^{*} \equiv \sum_{j=1}^{n} \lambda_{j}(t) \frac{\partial}{\partial \tau_{j}}+\sum_{i=1}^{m} \sum_{q_{i}=0}^{s_{i}-1} \lambda_{i}(t) \sigma_{i q_{i}} \frac{\partial}{\partial \sigma_{i q_{i}}}-A^{*}(t)
$$

Proof. Let $h^{(1)}(t, \tau, \sigma)=\sum_{k=1}^{n} \sum_{j=1}^{n} h_{k j}(t) c_{j}(t) e^{\tau_{k}}+\sum_{k=1}^{n} \sum_{i=1}^{m} \sum_{q_{i}=0}^{s_{i}-1} h_{k i q_{i}}(t) c_{k}(t) \sigma_{i q_{i}}$.

Determine solutions of system (3.4) in the form

$$
y^{(1)}(t, \tau, \sigma)=\sum_{k=1}^{n} \sum_{j=1}^{n} y_{k j}(t) c_{k}(t) e^{\tau_{j}}+\sum_{k=1}^{n} \sum_{i=1}^{m} \sum_{q_{i}=0}^{s_{i}-1} y_{k i q_{i}}(t) c_{k}(t) \sigma_{i q_{i}} .
$$


Substituting (3.8) in (3.4) and equating separately coefficients at $e^{\tau_{j}}$ and $\sigma_{i q_{i}}$, we obtain the equations

$$
\begin{gathered}
{\left[\lambda_{k}(t)-\lambda_{j}(t)\right] y_{k j}(t)=h_{k j}(t), \quad k, j=\overline{1, n}} \\
{\left[\lambda_{i}(t)-\lambda_{k}(t)\right] y_{i q_{i} k}(t)=h_{k i q_{i}}(t), \quad i=\overline{1, m}, \quad q_{i}=\overline{0, s_{i}-1}, k=\overline{1, n} .}
\end{gathered}
$$

One can see from this that obtained equations are solvable if and only if

$$
h_{k k}(t) \equiv 0, \quad k=\overline{1, n}, \quad h_{i i q_{i}}(t) \equiv 0, \quad i=\overline{1, m}, q_{i}=\overline{0, s_{i}-1}
$$

and these conditions coincide with conditions (3.6). Theorem 3.1 is proved.

Remark 3.2. Equations (1.1) imply that under conditions (3.6), system (3.4) has a solution in $U^{(1)}$ representable in the form

$$
\begin{aligned}
y^{(1)}(t, \tau, \sigma)= & \sum_{k=1}^{n} \sum_{j=1, j \neq k}^{n} \frac{h_{k j}(t)}{\left[\lambda_{k}(t)-\lambda_{j}(t)\right]} c_{j}(t) e^{\tau_{j}}+\sum_{k=1}^{n} \alpha_{k}(t) c_{k}(t) e^{\tau_{k}} \\
& +\sum_{i=1}^{m} \sum_{q_{i}=0}^{s_{i}-1} \gamma_{i q_{i}}(t) c_{i}(t) \sigma_{i q_{i}}+\sum_{k=1}^{n} \sum_{i=1, i \neq k}^{m} \sum_{q_{i}=0}^{s_{i}-1} \frac{h_{k i q_{i}}(t)}{\left[\lambda_{i}(t)-\lambda_{k}(t)\right]} c_{k}(t) \sigma_{i q_{i}},
\end{aligned}
$$

where $\alpha_{k}(t), \gamma_{i q_{i}}(t) \in C^{\infty}\left([0, T], C^{1}\right)$ are arbitrary functions.

Consider now system (3.5). As det $A(t) \equiv 0$ in points $t=t_{i}, i=\overline{1, m}$, this system does not always have a solution in $U^{(0)}$. Introduce the space $V^{(0)} \subset U^{(0)}$ consisting of vector functions

$$
z^{(0)}(t)=\sum_{j=1}^{n} z_{j}(t) c_{j}(t), \quad z_{j}(t) \in C^{\infty}\left([0, T], C^{1}\right), j=\overline{1, n},
$$

having the properties

$$
\left[D^{l_{i}}\left(z^{(0)}(t), d_{i}(t)\right)\right]_{t=t_{i}}=\left(D^{l_{i}} z_{i}\right)\left(t_{i}\right)=0, \quad \forall l_{i}=\overline{0, s_{i}-1}, i=\overline{1, m}
$$

where $d_{i}(t)$ are eigenvectors of the operator $A^{*}(t)$ with regard to eigenvalues $\bar{\lambda}_{i}(t), i=\overline{1, m}$. Let $h^{(0)}(t)=\sum_{j=1}^{n} h_{j}(t) c_{j}(t) \in V^{(0)}$, that is,

$$
\left(D^{l_{i}} h_{i}\right)\left(t_{i}\right)=0 \quad \forall l_{i}=\overline{0, s_{i}-1}, i=\overline{1, m}
$$

Determine a solution of system (3.5) in the

$$
y^{(0)}(t)=\sum_{j=1}^{n} y_{j}(t) c_{j}(t)
$$


Substituting this function in (3.5), we obtain

$$
-\sum_{j=1}^{n} y_{j}(t) \lambda_{j}(t) c_{j}(t)=\sum_{j=1}^{n} h_{j}(t) c_{j}(t) .
$$

Since $\left\{c_{j}(t)\right\}$ is a basis in $C^{n}$, we get

$$
\begin{array}{r}
-\lambda_{i}(t) y_{i}(t)=h_{i}(t), \quad i=\overline{1, m}, \\
-\lambda_{j}(t) y_{j}(t)=h_{j}(t), \quad j=\overline{m+1, n} .
\end{array}
$$

It is easy to see that (3.18) has the unique solution

$$
y_{j}(t)=\frac{-h_{j}(t)}{\lambda_{j}(t)}, \quad j=\overline{m+1, n}
$$

By virtue of conditions (3.14), the function $h_{i}(t)$ can be represented in the form

$$
h_{i}(t)=\left(t-t_{i}\right)^{s_{i}} \widehat{h}_{i}(t), \quad i=\overline{1, m},
$$

where $\widehat{h}_{i}(t) \in C^{\infty}\left([0, T], C^{1}\right.$ is the certain scalar function, $-\left(t-t_{i}\right)^{S_{i}} k_{i}(t) y_{i}(t)=\left(t-t_{i}\right)^{s_{i}} \widehat{h}_{i}(t)$, and we see that

$$
y_{i}(t)= \begin{cases}\frac{-\widehat{h}_{i}(t)}{k_{i}(t)}, & t \neq t_{i} \\ \gamma_{i}, & t=t_{i}\end{cases}
$$

where $\gamma_{i}$ are arbitrary constants, $i=\overline{1, m}$. However, the solution of system (3.5) should belong to the space $U^{(0)}$, and it means that $y_{i}(t) \in C^{\infty}\left([0, T], C^{1}\right)$. Therefore, constants in (3.21) $\gamma_{i}=$ $\left.\left(\widehat{h}_{i}(t) / k_{i}(t)\right)\right|_{t=t_{i}}$ and functions are determined uniquely in the form

$$
y_{i}(t)=\frac{-\widehat{h}_{i}(t)}{k_{i}(t)}, \quad \forall t \in[0, T], i=\overline{1, m}
$$

Thus, under conditions (3.14), system (3.5) has the solution $y^{(0)}(t)$ in $U^{(0)}$ of

$$
y^{(0)}(t)=-\sum_{i=1}^{m} \frac{\widehat{h}_{i}(t)}{k_{i}(t)} c_{i}(t)-\sum_{j=m+1}^{n} \frac{h_{i}(t)}{\lambda_{i}(t)} c_{i}(t),
$$

where $h_{i}(t)=\widehat{h}_{i}(t) /\left(t-t_{i}\right)^{s_{i}}$ (in points $t=t_{i}, i=\overline{1, m}$, this equality is understood in the limiting sense). We summarize received outcome in the form of the following assertion.

Theorem 3.3. Let the operator $A(t)$ satisfy condition (i), and let its spectrum satisfy conditions (ii)(iv). Then, for any vector function $h^{(0)}(t) \in V^{(0)}$, system (3.5) has the unique solution $y^{(0)}(t)$ in space $U^{(0)}$. 
For uniquely determination of functions $\alpha_{j}(t), \gamma_{i q_{i}}(t)$, consider system (3.4) with additional conditions:

$$
\begin{gathered}
y^{(1)}(0,0,0)=y_{*} \\
\left\langle-\frac{\partial y^{(1)}}{\partial t}, v_{j}(t, \tau, \sigma)\right\rangle \equiv 0 \quad \forall t \in[0, T], j=\overline{1, n} \\
\left\langle-\frac{\partial y^{(1)}}{\partial t}, v_{i q_{i}}(t, \tau, \sigma)\right\rangle \equiv 0, \quad i=\overline{1, m}, q_{i}=\overline{0, s_{i}-1}
\end{gathered}
$$

where $y_{*} \in C^{n}$ is a constant vector.

We have the following result.

Theorem 3.4. Let conditions of Theorem 3.1 hold. Then, the system (3.4) with additional conditions (3.24)-(3.25) has solutions of the form (3.11) in which all summands are uniquely determinate except for $\gamma_{i q_{i}}(t) c_{i}(t) \sigma_{i q_{i}}\left(i=\overline{1, m}, q_{i}=\overline{0, s_{i}-1}\right)$. Functions $\gamma_{i q_{i}}(t)$ in the last summand are determined by the formula

$$
\gamma_{i q_{i}}(t)=\gamma_{i q_{i}}^{0} \cdot e^{P_{i q_{i}}(t)}+f_{i q_{i}}(t)
$$

where $P_{i q_{i}}(t), f_{i q_{i}}(t)$ are known functions, and $\gamma_{i q_{i}}^{0}$ arbitrary constants.

Proof. Denote in (3.11) that

$$
g_{k j}(t)=\frac{h_{k j}(t)}{\lambda_{j}(t)-\lambda_{k}(t)}, \quad g_{k i q_{i}}(t)=\frac{h_{k i q_{i}}(t)}{\lambda_{i}(t)-\lambda_{k}(t)} .
$$

Using (3.11) and condition (3.24), we obtain the equality

$$
\sum_{k=1}^{n} \sum_{j=1}^{n} g_{k j}(0) c_{j}(0)+\sum_{k=1}^{n} \alpha_{k}(0) c_{k}(0)=y_{*}
$$

Multiplying this equality scalarly by $d_{s}(0)$, we get

$$
\alpha_{s}(0)=\left(y_{*}, d_{s}(0)\right)-\sum_{k=1, k \neq s}^{n} g_{k s}(0) \equiv \alpha_{s}^{0}, \quad s=\overline{1, n} .
$$

By (3.11) and conditions (3.25), we have

$$
-\dot{\alpha}_{S}(t)-\left(\dot{c}_{s}(t), d_{s}(t)\right) \alpha_{S}(t)-\sum_{j=1, j \neq s}^{n} g_{s j}(t)\left(\dot{c}_{j}(t), d_{s}(t)\right)=0, \quad s=\overline{1, n} .
$$

Considering these equations with initial conditions (3.30), we can uniquely obtain functions $\alpha_{s}(t), s=\overline{1, n}$. 
Now, using (3.11) and conditions (3.26), we get

$$
-\dot{\gamma}_{i q_{i}}(t)-\left(\dot{c}_{i}(t), d_{i}(t)\right) \gamma_{i q_{i}}(t)-\sum_{k=1, k \neq i}^{n} g_{k i q_{i}}(t)\left(\dot{c}_{k}(t), d_{i}(t)\right)=0, \quad i=\overline{1, m}, q_{i}=\overline{0, s_{i}-1}
$$

This implies that $\gamma_{i q_{i}}(t)$ have the form (3.27) where

$$
\begin{gathered}
P_{i q_{i}}(t)=-\int_{t_{i}}^{t}\left(\dot{c}_{i}(s), d_{i}(s)\right) d s \\
f_{i q_{i}}(t)=e^{P_{i q_{i}}(t)} \int_{t_{i}}^{t} e^{-P_{i q_{i}}(s)} \sum_{k=1, k \neq i}^{n} g_{k i q_{i}}(s)\left(\dot{c}_{k}(s), d_{i}(s)\right) d s .
\end{gathered}
$$

Theorem 3.4 is proved.

Remark 3.5. If conditions (3.6) hold for $h^{(1)}(t, \tau, \sigma) \in U^{(1)}$ and $h^{(0)}(t) \in U^{(0)}$, then system (3.3) has a solution in the space $U$, representable in the form of

$$
y(t, \tau, \sigma)=y^{(1)}(t, \tau, \sigma)+y^{(0)}(t)
$$

where $y^{(1)}(t, \tau, \sigma)$ is a function in the form of (3.11), and $y^{(0)}(t)$ is a function in the form of (3.23); moreover, functions $\alpha_{k}(t) \in C^{\infty}\left([0, T], C^{1}\right)$ are found uniquely in (3.11), and functions $\gamma_{i q_{i}}(t)$ are determined up to arbitrary constants $\gamma_{i q_{i}}^{0}$ in the form of (3.27).

Let us give the following result.

Theorem 3.6. Let $h^{(0)}(t) \in U^{(0)}, h^{(1)}(t, \tau, \sigma) \in U^{(1)}$, and conditions (i)-(iv), (3.6), (3.24)-(3.26) hold. Then, there exist unique numbers $\gamma_{i q_{i}}^{0}$ involved in (3.27), such that the function (3.34) satisfies the condition

$$
P y \equiv-\frac{\partial y^{(0)}}{\partial t}-\sum_{i=1}^{m} \sum_{q_{i}=0}^{s_{i}-1} \frac{\left(t-t_{i}\right)^{q_{i}}}{q_{i} !} \frac{\partial y^{(1)}}{\partial \sigma_{i q_{i}}}+H^{(0)}(t) \in V^{(0)}
$$

where $H^{(0)}(t) \in V^{(0)}$ is a fixed vector function.

Proof. To determine functions uniquely, calculate

$$
\begin{aligned}
P y \equiv & -\sum_{i=1}^{m}\left[\frac{h_{i}(t)}{k_{i}(t)} c_{i}(t)\right]^{\prime}-\sum_{j=m+1}^{n}\left[\frac{h_{j}(t)}{\lambda_{j}(t)} c_{j}(t)\right]^{\prime}-\sum_{i=1}^{m} \sum_{q_{i}=0}^{s_{i}-1} \frac{\left(t-t_{i}\right)^{q_{i}}}{q_{i} !} \gamma_{i q_{i}}(t) c_{i}(t) \\
& +\sum_{\substack{k=1 \\
i \neq 1}}^{n} \sum_{\substack{i \neq 1 \\
i \neq k}}^{m} \sum_{q_{i}=0}^{s_{i}-1} \frac{\left(t-t_{i}\right)^{q_{i}}}{q_{i} !} \cdot \frac{h_{i q_{i}}(t)}{\lambda_{i}(t)-\lambda_{k}(t)} c_{k}(t)+H^{(0)}(t),
\end{aligned}
$$


Abstract and Applied Analysis

$$
\begin{aligned}
\left(P y, d_{i}(t)\right) \equiv & -\left[\frac{h_{i}(t)}{k_{i}(t)}\right]^{\prime}-\sum_{i=1}^{m} \frac{h_{i}(t)}{k_{i}(t)}\left[\dot{c}_{i}(t), d_{i}(t)\right]-\sum_{j=m+1}^{n}\left[\frac{h_{j}(t)}{k_{j}(t)}\right]\left(\dot{c}_{j}(t), d_{i}(t)\right) \\
& -\sum_{q_{i}=0}^{s_{i}-1} \sum_{i=1}^{m} \frac{\left(t-t_{i}\right)^{q_{i}}}{q_{i} !} \gamma_{i q_{i}}(t)+\left[H^{(0)}(t), d_{i}(t)\right], \quad i=\overline{1, m} .
\end{aligned}
$$

Denote by $r_{i}(t)$ the known function

$$
r_{i}(t) \equiv-\left[\frac{h_{i}(t)}{k_{i}(t)}\right]^{\prime}-\sum_{i=1}^{m} \frac{h_{i}(t)}{k_{i}(t)}\left[\dot{c}_{i}(t), d_{i}(t)\right]-\sum_{j=m+1}^{n}\left[\frac{h_{j}(t)}{k_{j}(t)}\right]\left(\dot{c}_{j}(t), d_{i}(t)\right)+\left(H^{(0)}(t), d_{i}(t)\right),
$$

and write the conditions (3.13) for $\left(P y, d_{i}(t)\right)$. Taking into account expression (3.27) for $\gamma_{i q_{i}}(t)$, we get

$$
\sum_{q_{i}=0}^{s_{i}-1} \gamma_{i q_{i}}^{0}\left[D^{l_{i}}\left(\frac{\left(t-t_{i}\right)^{q_{i}}}{q_{i} !} e^{P_{i q_{i}}(x)}\right)\right]_{t=t_{i}}+\sum_{q_{i}=0}^{s_{i}-1}\left[D^{l_{i}} f_{i q_{i}}(t)\right]_{t=t_{i}}=\left[D^{l_{i}} r_{i}(t)\right]_{t=t_{i}{ }^{\prime}} \quad i=\overline{1, m}, l_{i}=\overline{0, s_{i}-1}
$$

Using the Leibnitz formula, we obtain that

$$
\begin{gathered}
{\left[D^{l_{i}}\left(\frac{\left(t-t_{i}\right)^{q_{i}}}{q_{i} !} e^{P_{i q_{i}}(t)}\right)\right]_{t=t_{i}}=\left[\sum_{v=0}^{l_{i}} C_{l_{i}}^{v}\left(\frac{\left(t-t_{i}\right)^{q_{i}}}{q_{i} !}\right)^{(v)}\left(e^{P_{i q_{i}}(t)}\right)^{\left(l_{i}-v\right)}\right]_{t=t_{i}}} \\
\quad=\left[\sum_{v=0}^{q_{i}} C_{l_{i}}^{v}\left(\frac{\left(t-t_{i}\right)^{q_{i}}}{q_{i} !}\right)^{(v)}\left(e^{P_{i q_{i}}(t)}\right)^{\left(l_{i}-v\right)}\right]_{t=t_{i}}=C_{l_{i}}^{q_{i}}\left(e^{P_{i q_{i}}(t)}\right)_{t=t_{i}}^{\left(l_{i}-q_{i}\right)},
\end{gathered}
$$

for $l_{i} \geq q_{i}$

$$
\left[D^{l_{i}}\left(\frac{\left(t-t_{i}\right)^{q_{i}}}{q_{i} !} e^{P_{i q_{i}}(t)}\right)\right]_{t=t_{i}}=0
$$

for $0 \leq l_{i} \leq q_{i}$.

Therefore, previous equalities are written in the form of

$$
\sum_{q_{i}=0}^{s_{i}-1} r_{i q_{i}}^{0} C_{l_{i}}^{q_{i}}\left(e^{P_{i q_{i}}(t)}\right)_{t=t_{i}}^{\left(l_{i}-q_{i}\right)}=r_{i l_{i}}^{0} \quad\left(i=\overline{1, m}, l_{i}=\overline{0, s_{i}-1}\right)
$$


where

$$
\begin{aligned}
& r_{i l_{i}}^{0}=-\sum_{q_{i}=0}^{s_{i}-1}\left[D^{l_{i}} f_{i q_{i}}(t)\right]_{t=t_{i}}-\left[D^{l_{i}} r_{i}(t)\right]_{t=t_{i}{ }^{\prime}} \\
& \text { for } l_{i}=0 \text {, we get } r_{i 0}^{0} e^{P_{i q_{i}}\left(t_{i}\right)}=r_{i 0}^{0} ; \\
& \text { for } l_{i}=1 \text {, we get } r_{i 0}^{0} c_{1}^{0}\left[e^{P_{i q_{i}}(t)}\right]_{t=t_{i}}^{\prime}+\gamma_{i 1}^{0} e^{P_{i q_{i}}\left(t_{i}\right)}=r_{i 1}^{0} ; \\
& \vdots \\
& \text { for } l_{i}=s_{i}-1 \text {, we get } r_{i 0}^{0} c_{s_{i}-1}^{0}\left[e^{P_{i q_{i}}(t)}\right]_{t=t_{i}}^{s_{i}-1}+\cdots+\gamma_{i s_{i}-1}^{0} e^{P_{i q_{i}}\left(t_{i}\right)}=r_{i s_{i}-1}^{0} .
\end{aligned}
$$

We obtain from here sequentially the numbers $\gamma_{i 0}^{0}, \ldots, \gamma_{i s_{i}-1}^{0}$. Theorem 3.6 is proved.

Thus, if conditions (3.24)-(3.26), (3.35) hold, all summands of solution (3.11) are defined uniquely.

So, if $h^{(0)}(t) \in U^{(0)}, h^{(1)}(t, \tau, \sigma) \in U^{(1)}$, and conditions (3.6), (3.24)-(3.26), and (3.35) are valid, then the systems (3.4), (3.5) (and (3.3) together with them) are solvable uniquely in the class $U=U^{(1)} \oplus U^{(0)}$. Two sequential problems $\left(\varepsilon^{k}\right)$ and $\left(\varepsilon^{k+1}\right)$ are connected uniquely by conditions (3.23)-(3.25), (3.30); therefore, by virtue of Theorems 3.1-3.6, they are solvable uniquely in the space $U$.

\section{Asymptotical Character of Formal Solutions}

Let $y_{-1}(t, \tau, \sigma), \ldots, y_{k}(t, \tau, \sigma)$ be solutions of formal problems $\left(\varepsilon^{-1}\right), \ldots,\left(\varepsilon^{k}\right)$ in the space $U$, respectively. Compose the partial sum for series (2.4):

$$
S_{n}(t, \tau, \sigma)=\sum_{k=-1}^{n} \varepsilon^{k} y_{k}(t, \tau, \sigma)
$$

and take its restriction $y_{\varepsilon n}(t)=S_{n}(t, \varphi(t) / \varepsilon, \psi(t, \varepsilon))$.

We have the following result.

Theorem 4.1. Let conditions (i)-(v) hold. Then, for sufficiently small $\varepsilon\left(0 \leq \varepsilon \leq \varepsilon_{0}\right)$, the estimates

$$
\left\|y(t, \tau)-y_{\varepsilon n}(t)\right\|_{C[0, T]} \leq C_{n} \varepsilon^{n+1}, \quad n=-1,0,1, \ldots,
$$

hold. Here, $y(t, \varepsilon)$ is the exact solution of problem (1.1), and $y_{\varepsilon n}(t)$ is the states above restriction of the nth partial sum of series (2.4).

Proof. The restriction $y_{\varepsilon n}(t)$ of series (2.4) satisfies the initial condition $y_{\varepsilon n}(0)=y^{0}$ and system (1.1) up to terms containing $\varepsilon^{n+1}$, that is,

$$
\varepsilon \frac{d y_{\varepsilon n}(t)}{d t}=A(t) y_{\varepsilon n}(t)+\varepsilon^{n+1} R_{n}(t, \varepsilon)+h(t)
$$


where $R_{n}(t, s)$ is a known function satisfying the estimate

$$
\|R(t, \varepsilon)\|_{C[0, T]} \leq \bar{R}_{n}, \quad \bar{R}_{n} \text { - const. }
$$

Under conditions of Theorem 4.1 on the spectrum of the operator $A(t)$ for the fundamental matrix $Y(t, s, \varepsilon) \equiv Y(t, \varepsilon) Y^{-1}(t, \varepsilon)$ of the system $\varepsilon \dot{Y}=A(t) Y$, the estimate

$$
\|Y(t, s, \varepsilon)\| \leq \text { const } \quad \forall(t, \varepsilon) \in Q \equiv\{0 \leq s \leq t \leq T\}, \forall \varepsilon>0 \in\left[0, \varepsilon_{0}\right],
$$

is valid. Here, $\varepsilon_{0}>0$ - is sufficiently small. Now, write the equation

$$
\varepsilon \frac{d \Delta(t, \varepsilon)}{d t}=A(t) \Delta(t, \varepsilon)-\varepsilon^{n+1} R_{n}(t, \varepsilon), \quad \Delta(0, \varepsilon)=0,
$$

for the remainder term $\Delta(t, \varepsilon) \equiv y(t, \varepsilon)-y_{\varepsilon n}(t)$. We obtain from this equation that

$$
\Delta(t, \varepsilon)=-\varepsilon^{n} \int_{0}^{t} Y(x, s, \varepsilon) R_{n}(s, \varepsilon) d s,
$$

whence we get the estimate

$$
\|\Delta(t, \varepsilon)\|_{C[0, T]} \leq-\varepsilon^{n} \bar{R}_{n}
$$

where $\bar{R}_{n}=\max _{(t, s) \in Q}\|Y(t, s, \varepsilon)\| \cdot\left\|\bar{R}_{n}(t, s)\right\| \cdot T$. So, we obtain the estimate

$$
\left\|y(t, \varepsilon)-y_{\varepsilon n}(t)\right\|_{C[0, T]} \leq \varepsilon^{n} \bar{R}_{n}, \quad n=-1,0,1, \ldots
$$

Taking instead of $y_{\varepsilon n}(t)$ the partial sum

$$
y_{\varepsilon, n+1}(t) \equiv y_{\varepsilon n}(t)+\varepsilon^{n+1} y_{n+1}\left(t, \frac{\varphi(t)}{\varepsilon}, \psi(t, \varepsilon)\right)
$$

we get

$$
\left\|\left(y(t, \varepsilon)-y_{\varepsilon n}(t)\right)-\varepsilon^{n+1} y_{n+1}\left(t, \frac{\varphi(t)}{\varepsilon}, \psi(t, \varepsilon)\right)\right\| \leq \varepsilon^{n+1} \bar{R}_{n+1},
$$

which implies the estimates (4.2). Theorem 4.1 is proved. 


\section{Example}

Let it be required to construct the asymptotical solution for the Cauchy problem

$$
\varepsilon\left(\begin{array}{l}
\dot{y} \\
\dot{z}
\end{array}\right)=\left(\begin{array}{cc}
-5 t^{2}+4 & 2 t^{2}-2 \\
-10 t^{2}+10 & 4 t^{2}-5
\end{array}\right)\left(\begin{array}{c}
y \\
z
\end{array}\right)+\left(\begin{array}{c}
t^{2} h_{1}(t) \\
0
\end{array}\right), \quad y(0, \varepsilon)=y^{0}, z(0, \varepsilon)=z^{0}
$$

where $h_{1}(t) \in C^{\infty}[0,2], \varepsilon>0$ is a small parameter. Eigenvalues of the matrix $A(t)=$ $\left(\begin{array}{cc}-5 t^{2}+4 & 2 t^{2}-2 \\ -10 t^{2}+10 & 4 t^{2}-5\end{array}\right)$ are $\lambda_{1}(t)=-t^{2}, \lambda_{2}(t)=-1$. Eigenvectors of matrices $A(t)$ and $A^{*}(t)$, are, respectively,

$$
\varphi_{1}=\left(\begin{array}{l}
1 \\
2
\end{array}\right), \quad \varphi_{2}=\left(\begin{array}{l}
2 \\
5
\end{array}\right), \quad \psi_{1}=\left(\begin{array}{c}
5 \\
-2
\end{array}\right), \quad \psi_{2}=\left(\begin{array}{c}
-2 \\
1
\end{array}\right)
$$

We get $\left(h(t), \psi_{1}(t)\right) \equiv 5 t^{2} h_{1}(t)$. Therefore,

$$
\left(h(0), \psi_{1}(0)\right)=0, \quad \frac{d}{d t}\left(h(0), \psi_{1}(0)\right)=0 .
$$

Hence, we can apply to problem (5.1) the above developed algorithm of the regularization method.

At first, obtain the basic Lagrange-Silvestre polynomials $K_{j i}(t)$. Since $\psi(t) \equiv \lambda_{1}(t)=$ $-t^{2}$, there will be two such polynomials: $K_{00}(t)$ and $K_{01}(t)$.

Take the arbitrary numbers $a_{00}(t)$ and $a_{01}(t)$, and set the interpolation conditions for the polynomial $r(t)$,

$$
r(t)=a_{00}, \quad \dot{r}(1)=a_{01} .
$$

Expand $r(t)$ onto partial fractions

$$
\frac{r(t)}{\psi(t)}=\frac{A}{t^{2}}+\frac{B}{t}
$$

from where

$$
r(t) \equiv A+B t
$$

Use the interpolation polynomial (5.4). We get $A=a_{00}, B=a_{01}$. Hence, (5.6) takes the form

$$
r(t) \equiv a_{00}+t a_{01}
$$

Since numbers $a_{00}$ and $a_{01}$ are arbitrary, basic Lagrange-Silvestre polynomials will be coefficients standing before them, that is,

$$
K_{00}(t) \equiv 1, \quad K_{01}(t) \equiv t .
$$


Introduce the regularizing variables

$$
\begin{aligned}
\sigma_{00} & =e^{(1 / \varepsilon) \int_{0}^{t} \lambda_{1} d s} \int_{0}^{t} e^{-(1 / \varepsilon) \int_{0}^{s} \lambda_{1} d x} \cdot K_{00}(s) d s=e^{-t^{3} / 3 \varepsilon} \int_{0}^{t} e^{s^{3} / 3 \varepsilon} d s \equiv p_{00}(t), \\
\sigma_{01} & =e^{(1 / \varepsilon) \int_{0}^{t} \lambda_{1} d s} \int_{0}^{t} e^{-(1 / \varepsilon) \int_{0}^{s} \lambda_{1} d x} \cdot K_{01}(s) d s=e^{-t^{3} / 3 \varepsilon} \int_{0}^{t} e^{s^{3} / 3 \varepsilon} \cdot s d s \equiv p_{01}(t), \\
\tau_{1} & =\frac{1}{\varepsilon} \int_{0}^{t} \lambda_{1} d s=-\frac{t^{3}}{3 \varepsilon} \equiv q_{1}(t), \quad \tau_{2}=\frac{1}{\varepsilon} \int_{0}^{t} \lambda_{2} d s=-\frac{t}{\varepsilon} \equiv q_{2}(t) .
\end{aligned}
$$

Construct the extended problem corresponding to problem (5.1):

$$
\begin{aligned}
\varepsilon \frac{\partial w}{\partial t} & +\lambda_{1}(t) \frac{\partial w}{\partial \tau_{1}}+\lambda_{2}(t) \frac{\partial w}{\partial \tau_{2}}+\lambda_{1}(t) \sigma_{00} \frac{\partial w}{\partial \sigma_{00}}+\lambda_{1}(t) \sigma_{01} \frac{\partial w}{\partial \sigma_{01}}+\varepsilon \frac{\partial w}{\partial \sigma_{00}}+\varepsilon t \frac{\partial w}{\partial \sigma_{01}}-A(t) w \\
& =h(t), \quad w(0,0,0, \varepsilon)=w^{0},
\end{aligned}
$$

where $\tau \equiv\left(\tau_{1}, \tau_{2}\right), \sigma=\left(\sigma_{00}, \sigma_{01}\right), w=w(t, \tau, \sigma, \varepsilon)$.

Determining solutions of problem (5.10) in the form of a series

$$
w(t, \tau, \sigma, \varepsilon)=\sum_{k=0}^{\infty} \varepsilon^{k} w_{k}(t, \tau, \sigma),
$$

we obtain the following iteration problems:

$$
\begin{aligned}
& L w_{0} \equiv \lambda_{1}(t)\left[\frac{\partial w_{0}}{\partial \tau}+\frac{\partial w_{0}}{\partial \sigma_{00}}+t \cdot \sigma_{01} \frac{\partial w_{0}}{\partial \sigma_{01}}\right]+\lambda_{2}(t) \frac{\partial w_{0}}{\partial \tau_{2}}-A(t) w_{0}=h(t), \quad w_{0}(0,0,0)=w^{0} \\
& L w_{1}=-\frac{\partial w_{0}}{\partial t}-\frac{\partial w_{0}}{\partial \sigma_{00}}-t \frac{\partial w_{0}}{\partial \sigma_{01}}, \quad w_{1}(0,0,0)=0 \\
& \vdots
\end{aligned}
$$

We determine solutions of iteration problems (5.12), (5.13), and so on in the space $U$ of functions in the form of

$$
\begin{aligned}
& w(t, \tau, \sigma)=w_{1}(t) e^{\tau_{1}}+w_{2}(t) e^{\tau_{2}}+w_{00}(t) \sigma_{00}+w_{01}(t) \sigma_{01}+w_{0}(t), \\
& w_{0}(t), w_{1}(t), w_{2}(t), w_{00}(t), w_{01}(t) \in C^{\infty}\left([0,2], C^{2}\right) .
\end{aligned}
$$

Directly calculating, we obtain the solution of system (5.12) in the form of

$$
w_{0}(t, \tau, \sigma)=\alpha_{1}(t) \varphi_{1} e^{\tau_{1}}+\alpha_{2}(t) \varphi_{2} e^{\tau_{2}}+\gamma_{00}(t) \varphi_{1} \sigma_{00}+\gamma_{01}(t) \varphi_{1} \sigma_{01}+5 h_{1}(t) \varphi_{1}-2 t^{2} h_{1}(t) \varphi_{2}
$$

where $\alpha_{j}(t), \gamma_{j i}(t) \in C^{\infty}[0,2]$ are for now arbitrary functions. 
To calculate the functions $\alpha_{j}(t)$ and $\gamma_{i j}(t)$, we pass to the following iteration problem (5.13). Taking into account (5.15), it will be written in the form of

$$
\begin{aligned}
L w_{1}= & -\dot{\alpha}_{1}(t) \varphi_{1} e^{\tau_{1}}-\dot{\alpha}_{2}(t) \varphi_{2} e^{\tau_{2}}-\dot{\gamma}_{00}(t) \varphi_{1} \sigma_{00}-\dot{\gamma}_{01}(t) \varphi_{1} \sigma_{01} \\
& -5 \dot{h}_{1}(t) \varphi_{1}-\left(2 t^{2} h_{1}(t)\right)^{\prime} \varphi_{2}-\gamma_{00}(t) \varphi_{1}-t \gamma_{01}(t) \varphi_{1} .
\end{aligned}
$$

For solvability of problem (5.13) in the space $U$, it is necessary and sufficient to fulfill the conditions

$$
\begin{gathered}
-\dot{\alpha}_{1}(t)=0, \quad-\dot{\alpha}_{2}(t)=0, \quad-\dot{\gamma}_{00}(t)=0, \quad-\dot{\gamma}_{01}(t)=0, \\
-5 \dot{h}_{1}(0)-\gamma_{00}(0)=0, \quad-5 \ddot{h}_{1}(0)-\dot{\gamma}_{00}(0)-\gamma_{01}(0)=0 .
\end{gathered}
$$

Using solution (5.15) and the initial condition $w_{0}(0,0,0)=w^{0}$, we obtain the equation

$$
\alpha_{1}(0) \varphi_{1}+\alpha_{2}(0) \varphi_{2}+5 h_{1}(0) \varphi_{1}=w^{0} .
$$

Multiplying it (scalar) on $\psi_{1}$ and $\psi_{2}$, we obtain the values

$$
\begin{gathered}
\alpha_{1}(0)=\left(w^{0}, \psi_{1}\right)-5 h_{1}(0) \equiv 5 y^{0}-2 z^{0}-5 h_{1}(0), \\
\alpha_{2}(0)=\left(w^{0}, \psi_{2}\right)=z^{0}-2 y^{0} .
\end{gathered}
$$

Using equalities (5.17), and also the initial data (5.19), we obtain uniquely the functions $\alpha_{j}(t)$ and $\gamma_{j i}(t)$ :

$$
\begin{gathered}
\alpha_{1}(t)=5 y^{0}-2 z^{0}-5 h_{1}(0), \quad \alpha_{2}(t)=z^{0}-2 y^{0} \\
\gamma_{00}(t)=-5 \dot{h}_{1}(0), \quad \gamma_{01}(t)=-5 \ddot{h}_{1}(0) .
\end{gathered}
$$

Substituting these functions into (5.15), we obtain uniquely the solution of problem (5.12) in the space $U$,

$$
\begin{aligned}
w_{0}(t, \tau, \sigma)= & \left(5 y^{0}-2 z^{0}-5 h_{1}(0)\right) \varphi_{1} e^{\tau_{1}}+\left(z^{0}-2 y^{0}\right) \varphi_{2} e^{\tau_{2}} \\
& -5 \dot{h}_{1}(0) \varphi_{1} \sigma_{00}-5 \ddot{h}_{1}(0) \varphi_{1} \sigma_{01}+5 h_{1}(t) \varphi_{1}-2 t^{2} h_{1}(t) \varphi_{2} .
\end{aligned}
$$


Producing here restriction on the functions $\tau=q(t), \sigma=p(t)$, we obtain the principal term of the asymptotics for the solution of problem (5.1):

$$
\begin{aligned}
w_{0 \varepsilon}(t)= & \left(5 y^{0}-2 z^{0}-5 h_{1}(0)\right) \varphi_{1} e^{-t^{3} / 3 \varepsilon}+\left(z^{0}-2 y^{0}\right) \varphi_{2} e^{-t / \varepsilon} \\
& -5 \dot{h}_{1}(0) \varphi_{1} e^{-t^{3} / 3 \varepsilon} \int_{0}^{t} e^{s^{3} / 3 \varepsilon} d s-5 \ddot{h}_{1}(0) \varphi_{1} e^{-t^{3} / 3 \varepsilon} \int_{0}^{t} e^{s^{3} / 3 \varepsilon} s d s+5 h_{1}(t) \varphi_{1} \\
& -2 t^{2} h_{1}(t) \varphi_{2} .
\end{aligned}
$$

The zero-order asymptotical solution is obtained: it satisfies the estimate

$$
\left\|w(t, \varepsilon)-w_{0 \varepsilon}(t)\right\|_{C[0,2]} \leq C_{1} \cdot \varepsilon,
$$

where $w(t, \varepsilon)$ is an exact solution of problem (1.1), and $C_{1}>0$ is a constant independent of $\varepsilon$ at sufficiently small $\varepsilon\left(0<\varepsilon \leq \varepsilon_{0}\right)$.

\section{References}

[1] S. A. Lomov, Introduction to the General Theory of Singular Perturbations, vol. 112 of Translations of Mathematical Monographs, American Mathematical Society, Providence, RI, USA, 1992.

[2] L. A. Skinner, "Matched expansion solutions of the first-order turning point problem," SIAM Journal on Mathematical Analysis, vol. 25, no. 5, pp. 1402-1411, 1994.

[3] A. B. Vasil'eva, "On contrast structures of step type for a system of singularly perturbed equations," Computational Mathematics and Mathematical Physics, vol. 34, no. 10, pp. 1215-1223, 1994.

[4] A. B. Vasil'eva, "Contrast structures of step-like type for a second-order singularly perturbed quasilinear differential equation," Computational Mathematics and Mathematical Physics, vol. 35, no. 4, pp. 411-419, 1995.

[5] A. B. Vasil'eva, V. F. Butuzov, and L. V. Kalachev, The Boundary Function Method for Singular Perturbation Problems, vol. 14, Society for Industrial and Applied Mathematics, Philadelphia, Pa, USA, 1995.

[6] A. G. Eliseev and S. A. Lomov, "The theory of singular perturbations in the case of spectral singularities of a limit operator," Matematicheskiu Sbornik, vol. 131, no. 4, pp. 544-557, 1986.

[7] A. Ashyralyev, "On uniform difference schemes of a high order of approximation for evolution equations with a small parameter," Numerical Functional Analysis and Optimization, vol. 10, no. 5-6, pp. 593-606, 1989.

[8] G. I. Shishkin, J. J. H. Miller, and E. O'Riordan, Fitted Numerical Methods for Singular Perturbation Problems, Error Estimates in the Maximum Norm for Linear Problems in One and Two Dimensions, World Scientific, River Edge, NJ, USA, 1996.

[9] A. Ashyralyev and H. O. Fattorini, "On uniform difference schemes for second-order singular perturbation problems in Banach spaces," SIAM Journal on Mathematical Analysis, vol. 23, no. 1, pp. 29-54, 1992.

[10] A. Ashyralyev and P. E. Sobolevskii, New Difference Schemes for Partial Differential Equations, vol. 148 of Operator Theory: Advances and Applications, Birkhäuser, Basel, Switzerland, 2004.

[11] A. Ashyralyev and Y. Sözen, "A note on the parabolic equation with an arbitrary parameter at the derivative," Mathematical and Computer Modelling, vol. 54, no. 11-12, pp. 2565-2572, 2011.

[12] A. Ashyralyev, "On uniform difference schemes of a higher order of approximation for elliptical equations with a small parameter," Applicable Analysis, vol. 36, no. 3-4, pp. 211-220, 1990.

[13] A. Ashyralyev and H. O. Fattorini, "On difference schemes of the high order of accuracy for singular perturbation elliptic equations," in Investigation of Theory and Approximation Methods for Differential Equations, pp. 80-83, Ashgabat, Turkmenistan, 1991.

[14] A. Xu and Z. Cen, "Asymptotic behaviors of intermediate points in the remainder of the EulerMaclaurin formula," Abstract and Applied Analysis, vol. 2010, Article ID 134392, 8 pages, 2010. 
[15] M. De la Sen, "Asymptotic comparison of the solutions of linear time-delay systems with point and distributed lags with those of their limiting equations," Abstract and Applied Analysis, vol. 2009, Article ID 216746, 37 pages, 2009.

[16] F. Wang and A. Yukun, "Positive solutions for singular complementary Lid-stone boundary value problems," Abstract and Applied Analysis, vol. 2011, Article ID 714728, 13 pages, 2011.

[17] A. B. Vasile'va and L. V. Kalachev, "Singularly perturbed periodic parabolic equations with alternating boundary layer type solutions," Abstract and Applied Analysis, vol. 2010, Article ID 52856, 21 pages, 2006.

[18] H. Šamajová and E. Špániková, "On asymptotic behaviour of solutions to $n$-dimensional systems of neutral differential equations," Abstract and Applied Analysis, vol. 2011, Article ID 791323, 19 pages, 2011.

[19] I. Gavrea and M. Ivan, "Asymptotic behaviour of the iterates of positive linear operators," Abstract and Applied Analysis, vol. 2011, Article ID 670509, 11 pages, 2011. 


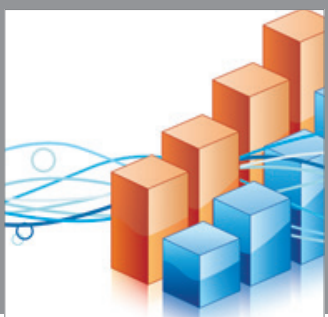

Advances in

Operations Research

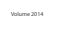

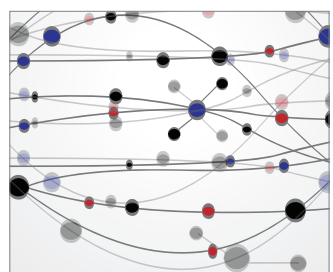

\section{The Scientific} World Journal
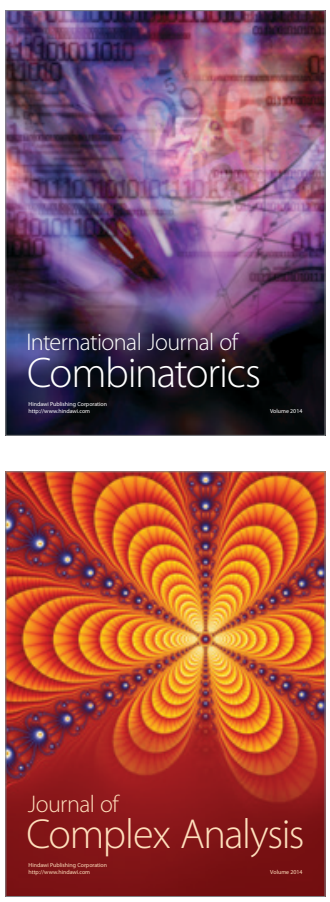

International Journal of

Mathematics and

Mathematical

Sciences
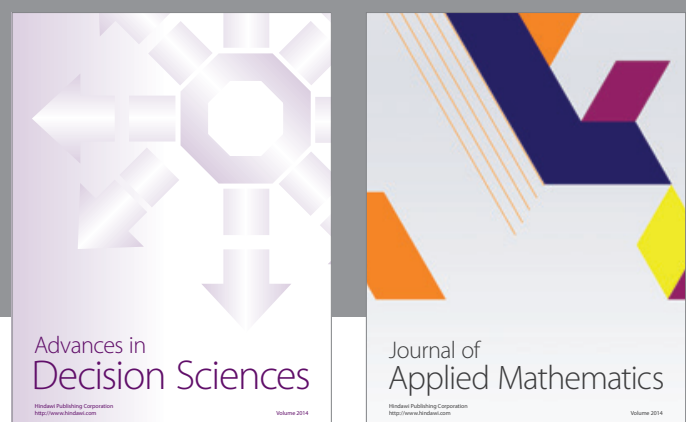

Journal of

Applied Mathematics
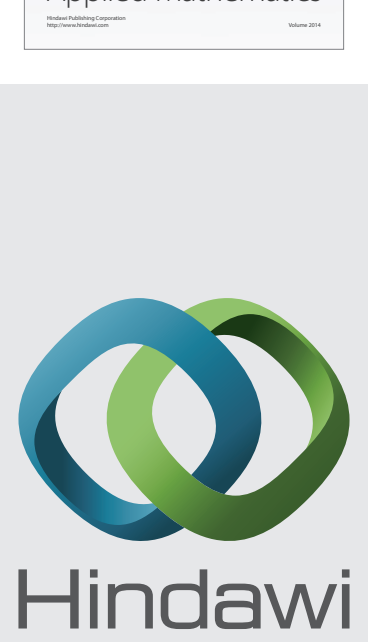

Submit your manuscripts at http://www.hindawi.com
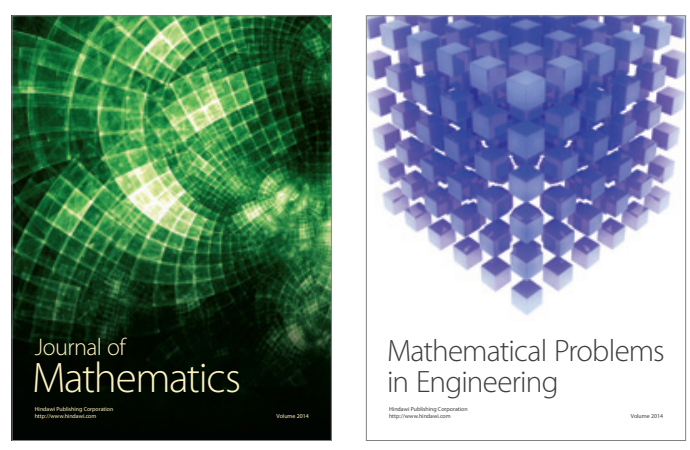

Mathematical Problems in Engineering
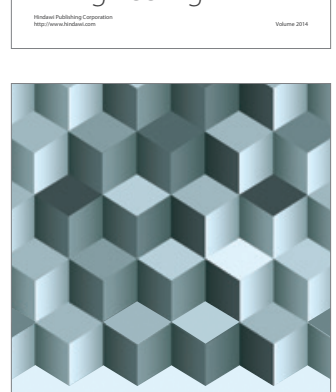

Journal of

Function Spaces
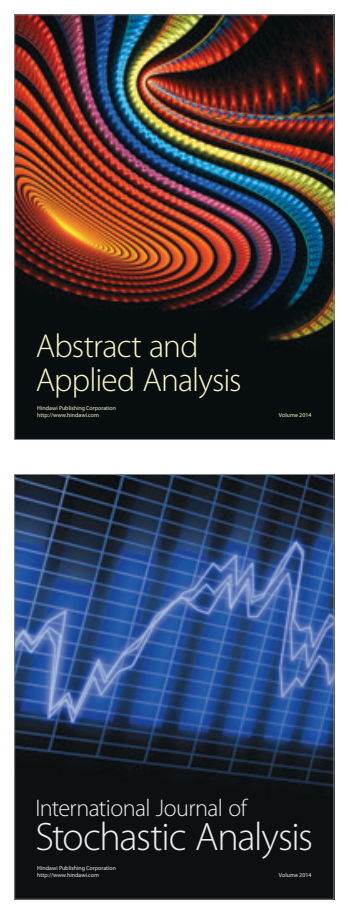

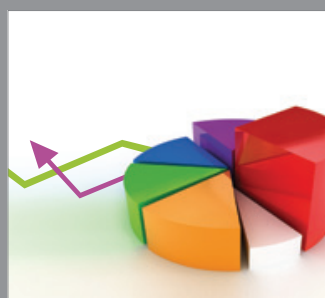

ournal of

Probability and Statistics

Promensencen
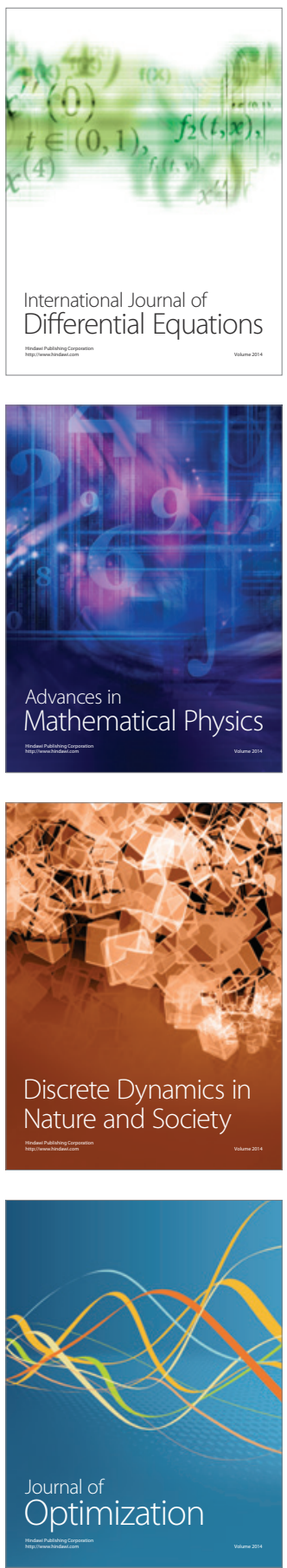